\title{
Optimal control problems with delay, the maximum principle and necessary conditions
}

\author{
J. F. FRANKENA \\ Dept. of Applied Mathematics, Twente University of Technology, Enschede, the Netherlands
}

(Received June 7, 1974)

\section{SUMMARY}

In this paper we consider a rather general optimal control problem involving ordinary differential equations with delayed arguments and a set of equality and inequality restrictions on state- and control variables. For this problem a maximum principle is given in pointwise form, using variational techniques. From this maximum principle necessary conditions are derived, as well as a Lagrange-like multiplier rule. Details may be found in ref. [2], together with extensions to the Hamilton-Jacobi equation and free end point problems.

\section{Introduction}

Recently the theory of optimal control problems has been developed into several directions. Concerning problems in which a given integral has to be minimized under restrictions ((in-) equality restrictions and differential equations) the introduction of delays in the independent variable can be mentioned, as well as the generalization to restrictions on both the state- and control variables.

Among others, Halanay [3], Hughes [6], [7] Pontryagin [9] and Sabbagh [10] have treated variational and optimal control problems with delays. On the other hand, Timman [11] and Nottrot [8], developed methods to treat problems with inequality restrictions on the stateand control variables.

The scope of this paper is to bridge both developments in a theory in which state variables and control variables are subjected to restrictions and in which a single constant delay occurs. The treatment of the inequality restrictions is in many respects similar to that given by Nottrot. The occurrence of a delay however requires nontrivial modifications. It should be mentioned that the results of these chapters include those for problems without delays.

In this paper the maximum principle is derived for optimal control problems of a general (nonlinear) structure, involving a single time delay $\tau$ in both the state- and control variables and with restrictions on both types of variables. This maximum principle furnishes a starting point for the derivation of necessary conditions.

It is worth while to note that the restriction to one constant delay is not very essential and facilitates the reading considerably. It is not difficult to generalize the results obtained in this paper to problems which include:

- delays which are multiples of $\tau$, i.e. problems which involve arguments $t, t-\tau, t-2 \tau$, etc.;

- one nonconstant delay $\tau(y(t), t)$ depending on the state $y(t)$ of the system and on $t$; see e.g. Asher and Sebesta [1].

Moreover, an arbitrary number of nonconstant delays can be considered in the problem statement, as Halanay did in [3]. The restriction to one constant delay, however, will furnish essential information about the structure of the difficulties to be encountered in any generalization.

\section{Statement of the problem}

In the following $t$ will indicate an independent variable ("time"), $y$ is a vector valued function 


$$
y=\left(\begin{array}{c}
y^{1} \\
\vdots \\
y^{n}
\end{array}\right)
$$

$y^{i}(i=1, \ldots, n)$ are called the state variables and $v$ is the vector valued function

$$
v=\left(\begin{array}{c}
v^{1} \\
\vdots \\
v^{m}
\end{array}\right),
$$

$v^{k}(k=1, \ldots, m)$ are called the control variables.

Let $\left[T_{0}, T_{1}\right]$ be a time interval and $\tau$ a positive number less than $T_{1}-T_{0}$. Suppose that $\alpha^{i}(i=1, \ldots, n)$ and $\beta^{k}(k=1, \ldots, m)$ are given functions on $\left[T_{0}-\tau, T_{0}\right]$, which are at least twice piecewise continuously differentiable; $y$ and $v$ are defined on $\left[T_{0}-\tau, T_{1}\right]$.

Consider those continuous solutions $y=\left(y^{1}, \ldots, y^{n}\right)$ of the initial value problem

$$
\left.\begin{array}{l}
\frac{d y^{i}}{d t}=f^{i}(t, y(t), y(t-\tau), v(t), v(t-\tau)), \quad i=1, \ldots, n, \\
T_{0}<t<T_{1} ; \\
y^{i}(t)=\alpha^{i}(t), \quad i=1, \ldots, n, T_{0}-\tau \leqq t \leqq T_{0} ; \\
v^{k}(t)=\beta^{k}(t), \quad k=1, \ldots, m, T_{0}-\tau \leqq t \leqq T_{0},
\end{array}\right\}
$$

which for properly chosen $v^{1}, \ldots, v^{m}$ satisfy the fixed end point condition $y\left(T_{1}\right)=Y_{1}$ and which minimize the integral

$$
\int_{T_{0}}^{T_{1}} F(t, y(t), y(t-\tau), v(t), v(t-\tau)) d t
$$

subject to the restrictions

$$
\phi^{j}(t, y(t), y(t-\tau), v(t), v(t-\tau)) \leqq 0, \quad j=1, \ldots, r
$$

(which are regarded as restrictions on the control variables) and

$$
g^{k}(t, y(t)) \leqq 0, \quad k=1, \ldots, v,
$$

the state variables restrictions.

Such solutions will be called extremals. It is assumed that at least one extremal exists connecting the points $Y_{0}=y\left(T_{0}\right)=\alpha\left(T_{0}\right)$ and $Y_{1}$. We assume that $v+r \leqq m^{\star}$.

It is supposed that $f^{i}, F, \phi^{j}$ and $g^{k}$ are piecewise continuous functions of all arguments and that these functions have piecewise continuous partial derivatives of first and second order with respect to their $2 \mathrm{nd}, 3 \mathrm{rd}, 4$ th and 5 th arguments (which is sufficient for our purposes); moreover $\partial g^{k} / \partial t(k=1, \ldots, v)$ are supposed to be piecewise continuous too.

In general the control variables may have jump discontinuities at a number of points in the interval $\left(T_{0}, T_{1}\right)$. These discontinuities will cause so-called "corner points", i.e. points at which the derivatives of the (continuous) state variables show a jump.

Even if not stated explicitly any relation involving derivatives which is considered in the sequel is understood to be considered in (open) intervals in $\left[T_{0}, T_{1}\right]$ not containing corner points in its interior.

When dealing with retarded or advanced arguments we use the following notations

$t_{i}=t-i \tau, \quad i=0, \pm 1, \pm 2 ; \ldots ;$

$y_{i}(t)=y\left(t_{i}\right)=y(t-i \tau)$, so $y_{-i}(t)=y\left(t_{-i}\right)=y(t+i \tau)$,

$v_{i}(t)=v\left(t_{i}\right)=v(t-i \tau)$, etc.

\footnotetext{
* This assumption may be weakened if there are control restrictions which do not explicitly depend on $y(t)$ and
} $y(t-\tau)$. 
With respect to such arguments we define every function of $t$ to be identically zero outside $\left[T_{0}, T_{1}\right]$ unless specified otherwise (as in the case of $y(t)$ and $v(t)$, which are defined on $\left[T_{0}-\tau, T_{0}\right]$ by $(2.1))$.

For specified values of its arguments a restriction is called active on some open subinterval of $\left[T_{0}, T_{1}\right]$ if the equality sign holds on this subinterval.

Suppose that the interval $\left[T_{0}, T_{1}\right]$ can be partitioned into a finite number of subintervals $\Delta_{l}=\left[\tau_{l-1}, \tau_{l}\right)(l=1, \ldots, \lambda)$ with $T_{0}=\tau_{0}, T_{1}=\tau_{\lambda}$, such that on every interval $\Delta_{l}$ certain restrictions are active, whereas the other restrictions are not. Let

$$
\begin{array}{ll}
\phi^{j}\left(t, y_{0}(t), y_{1}(t), v_{0}(t), v_{1}(t)\right)=0, & j=1, \ldots, q(q \leqq m) ; \\
\phi^{j}\left(t, y_{0}(t), y_{1}(t), v_{0}(t), v_{1}(t)\right)<0, & j=q+1, \ldots, r \\
g^{k}\left(t, y_{0}(t)\right)=0, & k=1, \ldots, \mu ; \\
g^{k}\left(t, y_{0}(t)\right)<0, & k=\mu+1, \ldots, v .
\end{array}
$$

on some interval $\Delta_{l}$; clearly $q$ and $\mu$ depend on $l$.

We assume every interval $\Delta_{l}$ to be of length less than $\tau$. This is a rather formal assumption since it can easily be satisfied by choosing "dummy" partitioning points.

The active restrictions play an important role in the present theory since a variation of any of the arguments should not cause the restriction functions to become positive.

\section{Reformulation of the problem}

In the calculus of variations, necessary conditions for minimization problems are derived by considering variations of the state variables. In optimal control problems however, these variations are due to variations of the control variables. The latter variations should be chosen in such a way that the restrictions-especially the active ones-are not violated. Now the control variables (and hence its variations) occur explicitly in the control variable restrictions (2.3) but not in the state variable restrictions (2.4). Hence it is not possible to relate control variable variations and state variable restrictions directly. This difficulty can be circumvented by taking the total time derivative of $g^{k}(t, y(t))(k=1, \ldots, v)$ and by using the relations (2.1):

$$
\begin{aligned}
h^{k}\left(t, y_{0}(t), y_{1}(t), v_{0}(t), v_{1}(t)\right) & =\frac{d g^{k}}{d t}= \\
= & \frac{\partial g^{k}}{\partial t}+\sum_{i=1}^{n} \frac{\partial g^{k}}{\partial y^{i}} f^{i}\left(t, y_{0}(t), y_{1}(t), v_{0}(t), v_{1}(t)\right), \quad(k=1, \ldots, v) .
\end{aligned}
$$

The corresponding restrictions (2.4) are:

$$
\int_{t_{0}}^{t} h^{k}\left(s, y_{0}(s), y_{1}(s), v_{0}(s), v_{1}(s)\right) d s \leqq 0 \quad(k=1, \ldots, v) .
$$

Now a relationship between control variables and state variable restrictions has been introduced it is possible to consider all restrictions (see (2.5)) as auxiliary control variables:

This is made explicit by the following definition of the new controls $\eta_{0}^{1}, \ldots, \eta_{0}^{m}$ :

$$
\begin{array}{ll}
\eta_{0}^{j}+h^{j}=0, & j=1, \ldots, v, \\
\eta_{0}^{j+v}+\phi^{j}=0, & j=1, \ldots, r, \quad \text { for all } t \in \Delta_{l}, 1 \leqq l \leqq \lambda . \\
\eta_{0}^{j}=v^{j}, & j=v+r+1, \ldots, m,
\end{array}
$$

It is supposed that the Jacobian

$$
\frac{\partial\left(h^{1}, \ldots, h^{v} ; \phi^{1}, \ldots, \phi^{r}\right)}{\partial\left(v_{0}^{1}, \ldots, v_{0}^{m}\right)}
$$


has rank $v+r$ on every interval $\Delta_{l}$ and furthermore that the components $v^{1}, \ldots, v^{m}$ have been arranged in such a way that

$$
\frac{\partial\left(h^{1}, \ldots, h^{v} ; \phi^{1}, \ldots, \phi^{r}\right)}{\partial\left(v_{0}^{1}, \ldots, v_{0}^{v+r}\right)}
$$

is nonsingular. Then the relations (3.3) can be inverted:

$$
\begin{array}{ll}
v_{0}^{j}=v_{0}^{j}\left(t, y_{0}, y_{1}, \eta_{0}, v_{1}\right), & j=1, \ldots, v+r ; \\
v_{0}^{j}=\eta_{0}^{j}, & j=v+r+1, \ldots, m .
\end{array}
$$

Let us consider the first $v+r$ relations more closely.

By definition,

whereas

$$
\begin{aligned}
v_{1}^{j}(t) & =v_{0}^{j}\left(t-\tau, y_{0}(t-\tau), y_{1}(t-\tau), \eta_{0}(t-\tau), v_{1}(t-\tau)\right)= \\
& =v_{0}^{j}\left(t-\tau, y_{1}(t), y_{2}(t), \eta_{1}(t), v_{2}(t)\right)
\end{aligned}
$$

$$
\begin{aligned}
v_{2}^{j}(t) & =v_{0}^{j}\left(t-2 \tau, y_{0}(t-2 \tau), y_{1}(t-2 \tau), \eta_{0}(t-2 \tau), v_{1}(t-2 \tau)\right)= \\
& =v_{0}^{j}\left(t-2 \tau, y_{2}(t), y_{3}(t), \eta_{2}(t), v_{3}(t)\right),
\end{aligned}
$$

and so on, until we arrive at the initial functions

$$
\alpha^{i}(t)(i=1, \ldots, n) \text { and } \beta^{j}(t)(j=1, \ldots, m), t \in\left[T_{0}-\tau, T_{0}\right] .
$$

Therefore the functions $v_{0}^{j}$ in (3.4) may be considered as functions of $t, t-\tau, t-2 \tau, \ldots ; y_{0}(t)$, $y_{1}(t), y_{2}(t), \ldots ; \eta_{0}(t), \eta_{1}(t), \ldots$, the number of which depends on the position $t$ in the interval $\left[T_{0}, T_{1}\right]$. Hence we may write the relations (3.4) in the form

$$
v_{0}^{j}=v_{0}^{j}\left(t_{0}, t_{1}, \ldots ; y_{0}, y_{1}, \ldots ; \eta_{0}, \eta_{1}, \ldots\right), \quad j=1, \ldots, m,
$$

regardless the special form of these relationships for

$$
j=v+r+1, \ldots, m \text {. }
$$

Substituting the relations (3.5) we define:

$$
\begin{aligned}
& Q\left(t_{0}, t_{1}, \ldots ; y_{0}, y_{1}, \ldots ; \eta_{0}, \eta_{1}, \ldots\right)=F\left(t, y_{0}, y_{1}, v_{0}, v_{1}\right) ; \\
& q^{i}\left(t_{0}, t_{1}, \ldots ; y_{0}, y_{1}, \ldots ; \eta_{0}, \eta_{1}, \ldots\right)=f^{i}\left(t, y_{0}, y_{1}, v_{0}, v_{1}\right), \quad i=1, \ldots, n ;
\end{aligned}
$$

for all $t \in \Delta_{l}, 1 \leqq l \leqq \lambda$.

The problem stated in section 2 can now be reformulated as follows.

Determine the continuous solutions $y_{0}^{i}(i=1, \ldots, n)$ of

$$
\begin{aligned}
& \frac{d y_{0}^{i}}{d t}=q^{i}\left(t_{0}, t_{1}, \ldots ; y_{0}, y_{1}, \ldots ; \eta_{0}, \eta_{1}, \ldots\right) \\
& i=1, \ldots, n, \quad T_{0}<t<T_{1} ; \\
& y_{0}^{i}(t)=\alpha^{i}(t), \quad i=1, \ldots, n, \quad T_{0}-\tau \leqq t \leqq T_{0} ; \\
& \eta_{0}^{j}(t)=\beta^{j}(t), \quad j=1, \ldots, m, \quad T_{0}-\tau \leqq t \leqq T_{0},
\end{aligned}
$$

which for properly chosen $\eta_{0}, \eta_{1}, \ldots$ satisfy the end point condition $y\left(T_{1}\right)=Y_{1}$ and which minimize the integral

$$
\int_{T_{0}}^{T_{1}} Q\left(t_{0}, t_{1}, \ldots ; y_{0}, y_{1}, \ldots ; \eta_{0}, \eta_{1}, \ldots\right) d t
$$

subject to the restrictions

$$
\begin{array}{ll}
\int_{T_{0}}^{t} \eta_{0}^{k} d s \geqq 0, & k=1, \ldots, \nu(\operatorname{see}(3.2)) \\
\eta_{0}^{\nu+j} \geqq 0, & j=1, \ldots, r(\text { from }(2.3)) .
\end{array}
$$


Moreover, since $g^{j}$ should be nonpositive on the entire interval $\left[T_{0}, T_{1}\right]$ any variation

$$
\delta \eta_{0}^{j}(t)
$$

of the optimal control (i.e. a control corresponding to an extremal $y(t)$ ) has to satisfy the inequality restrictions which result from

$$
\int_{T_{0}}^{t} \delta h^{j} d s \leqq 0, \quad j=1, \ldots, \mu, t \in\left[T_{0}, T_{1}\right] ;
$$

i.e., when $t \in \Delta_{l}$ :

$$
\int_{T_{0}}^{t} \delta \eta_{0}^{j} d s \geqq 0, \quad j=1, \ldots, \mu
$$

( $\mu$ is the number of active state restrictions).

From now on suppose that $y(t)$ is an extremal and that $y_{1}(t), \ldots ; v_{0}(t), v_{1}(t), \ldots$ and/or $\eta_{0}(t), \eta_{1}(t), \ldots$ are the corresponding "optimal" functions. The integral (2.2) along an extremal $y$ will be denoted by $J[y]$. A variation $\delta \eta_{0}(t)$ of the optimal control function $\eta(t)$ will be called admissible if the following conditions are satisfied:

(a) $\delta \eta^{j}(t)=0, \quad j=1, \ldots, m, \quad T_{0}-\tau \leqq t \leqq T_{0}$;

(b) $\delta y^{i}(t)=0, \quad i=1, \ldots, n, \quad T_{0}-\tau \leqq t \leqq T_{0}$;

(c) $\delta y^{i}(t)$ is piecewise smooth and uniformly small on

$T_{0}<t \leqq T_{1}, \quad i=1, \ldots, n$,

i.e. for any prescribed $\varepsilon>0,\left|\delta y^{i}(t)\right|<\varepsilon, i=1, \ldots, n, T_{0}<t \leqq T_{1}$ (terms of order $O\left(\varepsilon^{2}\right)$ will be neglected);

(d) The restrictions

$$
\begin{aligned}
\phi^{j} \leqq 0, & j=1, \ldots, r, \\
g^{k} \leqq 0, & k=1, \ldots, v
\end{aligned}
$$

are satisfied by the varied variables;

$y_{0}+\delta y_{0}, y_{1}+\delta y_{1}, v_{0}+\delta v_{0}, v_{1}+\delta v_{1}$,

the variations $\delta y_{0}, \delta y_{1}, \delta v_{0}$ and $\delta v_{1}$ being caused by the variation $\delta \eta_{0}$.

In the next section an analysis will be given of the influence of admissible variations of $\eta_{0}$ upon the integral (3.8) in which $y(t)$ is supposed to be an extremal of the problem.

\section{The influence of admissible variations; the adjoint equations}

As mentioned in the preceding section it is supposed that $y(t)$ is an extremal of the problem (3.7)-(3.12) which means that

$$
J[y]=\int_{T_{0}}^{T_{1}} Q\left(t_{0}, t_{1}, \ldots ; y_{0}, y_{1}, \ldots ; \eta_{0}, \eta_{1}, \ldots\right) d t
$$

is a minimum value. With respect to local (uniformly small) variations of the state variable $y(t)$ induced by an admissible variation $\delta \eta_{u}$ it follows that the variation $D$ of the integral is

$$
\begin{aligned}
D=\delta \int_{T_{0}}^{T_{1}} Q\left(t_{0}, t_{1}, \ldots ; y_{0}, y_{1}, \ldots ; \eta_{0}, \eta_{1}, \ldots\right) d t= \\
=\int_{T_{0}}^{T_{1}} Q\left(t_{0}, t_{1}, \ldots ; y_{0}+\delta y_{0}, y_{1}+\delta y_{1}, \ldots ; \eta_{0}+\delta \eta_{0}, \eta_{1}+\delta \eta_{1}, \ldots\right)- \\
\quad-Q\left(t_{0}, t_{1}, \ldots ; y_{0}, y_{1}, \ldots ; \eta_{0}, \eta_{1}, \ldots\right) d t \geqq 0 .
\end{aligned}
$$

In [2] it is shown that, neglecting $O\left(\varepsilon^{2}\right)$-terms, we have 


$$
\begin{aligned}
D=\int_{T_{0}}^{T_{1}}\{ & Q\left(t_{0}, t_{1}, \ldots ; y_{0}+\delta y_{0}, y_{1}+\delta y_{1}, \ldots ; \eta_{0}+\delta \eta_{0}, \eta_{1}+\delta \eta_{1}, \ldots\right)- \\
& \left.-Q\left(t_{0}, t_{1}, \ldots ; y_{0}+\delta y_{0}, y_{1}+\delta y_{1}, \ldots ; \eta_{0}, \eta_{1}, \ldots\right)\right\} d t+ \\
& +\sum_{i=0}^{\kappa} \int_{T_{0}}^{T_{1}-i \tau} \sum_{j=1}^{n} \frac{\partial Q}{\partial y_{i}^{j}}[t+i \tau] \delta y_{0}^{j}(t) d t .
\end{aligned}
$$

We now introduce, formally, $n$ continuous functions on $\left[T_{0}, T_{1}\right]: p^{1}(t), \ldots, p^{n}(t)$ which are supposed to be continuously differentiable on $\left(T_{0}, T_{1}\right)$, possibly with the exception of corner points $\tau_{l}(1 \leqq l \leqq \lambda)$, the boundary points of the subintervals $\Delta_{l}$. This will be done by adding to the last term in (4.2) a sum of integrals of $0=d / d t\left(p^{j} \delta y_{0}^{j}\right)-p^{j} \delta \dot{y}_{0}^{j}-\dot{p}^{j} \delta y_{0}^{j}=d / d t\left(p^{j} \delta y_{0}^{j}\right)-$ $\dot{p}^{j} \delta y_{0}^{j}-p^{j} \delta q^{j}$ with $\delta q^{j}$ evaluated with respect to $\delta y_{i}^{k}$ (Here, $p^{j}$ is still undefined).

By standard methods (see [2], [8], [11]) this leads to

$$
\begin{aligned}
& \sum_{i=0}^{\kappa} \int_{T_{0}}^{T_{1}-i \tau} \sum_{j=1}^{n} \frac{\partial Q}{\partial y_{i}^{j}}[t+i \tau] \delta y_{0}^{j}(t) d t=\left[\sum_{j=1}^{n} p^{j} \delta y_{0}^{j}\right]_{T_{0}}^{T_{1}}- \\
& \quad-\int_{T_{0}}^{T_{1}} \sum_{j=1}^{n} \dot{p}^{j}(t) \delta y_{0}^{j}(t) d t-\int_{T_{0}}^{T_{1}} \sum_{j=1}^{n} p^{j}(t)\left\{q^{j}\left(t_{0}, t_{1}, \ldots ; y_{0}+\delta y_{0}, \ldots ; \eta_{0}+\delta \eta_{0}, \ldots\right)-\right. \\
& \left.\quad-q^{j}\left(t_{0}, t_{1}, \ldots ; y_{0}+\delta y_{0}, \ldots ; \eta_{0}, \eta_{1}, \ldots\right)\right\} d t+ \\
& \quad+\sum_{i=0}^{\kappa} \int_{T_{0}}^{T_{1}-i \tau} \sum_{j=1}^{n}\left\{\sum_{k=1}^{n} p^{k}(t+i \tau) \frac{\partial q^{k}}{\partial y_{i}^{j}}[t+i \tau]+\frac{\partial Q}{\partial y_{i}^{j}}[t+i \tau]\right\} \delta y_{0}^{j}(t) d t
\end{aligned}
$$

Substitution of this into (4.2) yields

$$
\begin{aligned}
D= & \int_{T_{0}}^{T_{1}}\left\{Q\left(t_{0}, t_{1}, \ldots ; y_{0}+\delta y_{0}, \ldots ; \eta_{0}+\delta \eta_{0}, \ldots\right)-\right. \\
& \left.\quad-Q\left(t_{0}, t_{1}, \ldots ; y_{0}+\delta y_{0}, \ldots ; \eta_{0}, \eta_{1}, \ldots\right)\right\} d t- \\
& -\int_{T_{0}}^{T_{1}} \sum_{j=1}^{n} p^{j}(t)\left\{q^{j}\left(t_{0}, t_{1}, \ldots ; y_{0}+\delta y_{0}, \ldots ; \eta_{0}+\delta \eta_{0}, \ldots\right)-\right. \\
& \left.\quad-q^{j}\left(t_{0}, t_{1}, \ldots ; y_{0}+\delta y_{0}, \ldots ; \eta_{0}, \eta_{1}, \ldots\right)\right\} d t+ \\
+ & {\left[\sum_{j=1}^{n} p^{j}(t) \delta y_{0}^{j}(t)\right]_{T_{0}}^{T_{1}}+\sum_{i=0}^{\kappa} \int_{T_{0}}^{T_{1}-i \tau} \sum_{j=1}^{n}\left[\frac{\partial Q}{\partial y_{i}^{j}}[t+i \tau] \delta y_{0}^{j}(t)-\right.}
\end{aligned}
$$

Due to the fact that all functions are by definition identically zero for $t>T_{1}$, the last integrals may formally be rewritten as follows (replacing $T_{1}-i \tau$ by $T_{1}$ ):

$$
\begin{aligned}
& \sum_{i=0}^{\kappa} \int_{T_{0}}^{T_{1}-i \tau} \sum_{j=1}^{n}\left[\frac{\partial Q}{\partial y_{i}^{j}}[t+i \tau]-\right.\left.\sum_{k=1}^{n} p^{k}(t+i \tau) \frac{\partial q^{k}}{\partial y_{i}^{j}}[t+i \tau]\right] \delta y_{0}^{j}(t) d t- \\
&-\int_{T_{0}}^{T_{1}} \sum_{j=1}^{n} \dot{p}^{j}(t) \delta y_{0}^{j}(t) d t=\int_{T_{0}}^{T_{1}} \sum_{j=1}^{n}\left[\sum _ { i } \left\{\frac{\partial Q}{\partial y_{i}^{j}}[t+i \tau]-\right.\right. \\
&\left.\left.-\sum_{k=1}^{n} p^{k}(t+i \tau) \frac{\partial q^{k}}{\partial y_{i}^{j}}[t+i \tau]\right\}-\dot{p}^{j}(t)\right] \delta y_{0}^{j}(t) d t,
\end{aligned}
$$

where the summation over $i$ is extended, in fact, to those value of $i$ for which $t+i \tau \leqq T_{1}$. Since the number of summands obviously depends on $t \in\left[T_{0}, T_{1}\right]$, the limits of summation are omitted.

We now define the functions $p^{j}(t)(j=1, \ldots, n)$ as solutions of the following differential equations: 


$$
\begin{aligned}
& \dot{p}^{j}(t)=\sum_{i}\left\{\frac{\partial Q}{\partial y_{i}^{j}}[t+i \tau]-\sum_{k=1}^{n} p^{k}(t+i \tau) \frac{\partial q^{k}}{\partial y_{i}^{j}}[t+i \tau]\right\} ; j=1, \ldots, n, \\
& t \in\left(T_{0}, T_{1}\right)
\end{aligned}
$$

except possibly for "corner points" (of $y^{j}(t)$ ), i.e. points where the derivatives in the right-hand side show jumps and points $T_{1}-i \tau$, where the number of summands is altered. In these points the solutions are matched in order to define them as continuous functions.

We shall call the equations (3.7) and (4.4) adjoint equations; the variables $p^{j}(t)(j=1, \ldots, n)$ will be called adjoint variables. They are solutions of a linear first-order system which is an ordinary (i.e. non-delayed) system on the interval $\left(T_{1}-\tau, T_{1}\right)$. It suffices, therefore, to specify the values of $p^{j}(t)(j=1, \ldots, n)$ at $t=T_{1}$, as will be done as follows.

With the foregoing definition of $p^{j}(t)(j=1, \ldots, n)$ all terms in (4.3) except for the first two integrals drop out and defining

$$
\begin{aligned}
& K\left(t_{0}, t_{1}, \ldots ; y_{0}, y_{1}, \ldots ; \eta_{0}, \eta_{1}, \ldots ; p\right)=-Q\left(t_{0}, t_{1}, \ldots ; y_{0}, y_{1}, \ldots ; \eta_{0}, \eta_{1}, \ldots\right)+ \\
&+\sum_{j=1}^{n} p^{j}(t) q^{j}\left(t_{0}, t_{1}, \ldots ; y_{0}, y_{1}, \ldots ; \eta_{0}, \eta_{1}, \ldots\right) ; \\
& \sum_{j=1}^{n} p^{j}\left(T_{1}\right) \delta y_{0}^{j}\left(T_{1}\right)=d J\left(T_{1}, Y_{1}\right) \approx \delta J
\end{aligned}
$$

we arrive at the inequality

$$
\begin{aligned}
D= & \int_{T_{0}}^{T_{1}}\left[-K\left(t_{0}, t_{1}, \ldots ; y_{0}+\delta y_{0}, y_{1}+\delta y_{1}, \ldots ; \eta_{0}+\delta \eta_{0}, \eta_{1}+\delta \eta_{1}, \ldots ; p\right)+\right. \\
& \left.+K\left(t_{0}, t_{1}, \ldots ; y_{0}+\delta y_{0}, y_{1}+\delta y_{1}, \ldots ; \eta_{0}, \eta_{1}, \ldots ; p\right)\right] d t+\delta J\left[Y_{1}\right] \geqq 0 .
\end{aligned}
$$

We shall call

$$
H\left(t, y_{0}(t), y_{1}(t), v_{0}(t), v_{1}(t) ; p(t)\right)=K\left(t_{0}, t_{1}, \ldots ; y_{0}, y_{1}, \ldots ; \eta_{0}, \eta_{1}, \ldots ; p\right)
$$

the Hamiltonian function or shortly Hamiltonian of the problem. Using this function the equations (4.4) can be written in the comprehensive form

$$
\dot{p}^{j}(t)=-\sum_{i} \frac{\partial K}{\partial y_{i}^{j}}[t+i \tau], \quad j=1, \ldots, n .
$$

Obviously, since $D$ is the difference between the integrals along an (arbitrarily but admissibly) varied curve and an extremal from $\left(T_{0}, Y_{0}\right)$ to $\left(T_{1}, Y_{1}\right)$ whereas $\delta J$ is the difference between the integrals along two extremals we have the inequality

$$
D \geqq \delta J\left[Y_{1}\right]
$$

and consequently (4.7) reduces to

$$
\begin{aligned}
\int_{T_{0}}^{T_{1}}\left[-K\left(t_{0}, t_{1}, \ldots ;\right.\right. & \left.y_{0}+\delta y_{0}, y_{1}+\delta y_{0}, \ldots ; \eta_{0}+\delta \eta_{0}, \eta_{1}+\delta \eta_{1}, \ldots ; p\right) \\
& \left.+K\left(t_{0}, t_{1}, \ldots ; y_{0}+\delta y_{0}, y_{1}+\delta y_{1}, \ldots ; \eta_{0}, \eta_{1}, \ldots ; p\right)\right] d t \geqq 0
\end{aligned}
$$

for all admissible variations $\delta \eta_{0}$.

In the next section a maximum principle will be derived from this inequality by the choice of a special admissible variation.

\section{The maximum principle}

In this section an inequality will be given which expresses that for an extremal of the problem (3.7)-(3.12) the Hamiltonian $K\left(t_{0}, t_{1}, \ldots ; y_{0}, y_{1}, \ldots ; \eta_{0}, \eta_{1} ; \ldots ; p\right)$ is, in a certain sense, "maximal" with respect to the control variables $\eta_{0}, \eta_{1}, \ldots$. This maximum principle is the most important result of the present investigation since all other necessary conditions are 
easily obtained from it. More familiar forms of the maximum principle will be given in section 6 .

Our maximum principle is a generalization of the well-known Pontryagin maximum principle. In fact, when there are no delays involved in the problem, our result is exactly the maximum principle with mixed restrictions as derived by Nottrot [8].

The starting point of the considerations is the inequality (4.9) which holds for all admissible variations $\delta \eta_{0}$, i.e. variations for which, among others, the inequality (3.11):

$$
\int_{T_{0}}^{t} \delta \eta_{0}^{j} d s \geqq 0 \quad(j=1, \ldots, \mu)
$$

should hold for all $t \in\left[T_{0}, T_{1}\right]$.

In [2] it has been shown that using the particular variation

$$
\delta \eta_{0}^{j}(t)=0, \quad j=1, \ldots, m \text {, outside }\left[\sigma_{l-1}-\delta, \sigma_{l-1}+\delta\right] \text { and }\left[\sigma_{l}-\delta, \sigma_{l}+\delta\right], \text { which }
$$

are intervals in $\Delta_{l}$;

$$
\begin{aligned}
& \delta \eta_{0}^{j}(t)=\left\{\begin{array}{ll}
\varepsilon^{j}>0, & j=1, \ldots, \mu ; \\
\theta_{1} \varepsilon^{j}, \varepsilon^{j}>0, & j=\mu+1, \ldots, v ; \\
\varepsilon^{j}>0, & j=v+1, \ldots, v+q ; \\
\theta_{2} \varepsilon^{j}, \varepsilon^{j}>0, & j=v+q+1, \ldots, v+r \\
\varepsilon^{j}, & j=v+r+1, \ldots, m ;
\end{array} \quad\left(\sigma_{l-1}-\delta \leqq t \leqq \sigma_{l-1}+\delta\right) ;\right. \\
& \delta \eta_{0}^{j}(t)=\left\{\begin{array}{ll}
\theta_{3} \varepsilon^{j}, & j=1, \ldots, \mu, \varepsilon^{j} \text { as above } ; \\
\theta_{4} \varepsilon^{j}, & j=\mu+1, \ldots, v, \varepsilon^{j} \text { as above } ; \\
0, & j=v+1, \ldots, v+q ; \\
0, & j=v+q+1, \ldots, v+r ; \\
0, & j=v+r+1, \ldots, m ;
\end{array} \quad\left(\sigma_{l}-\delta \leqq t \leqq \sigma_{l}+\delta\right) ;\right.
\end{aligned}
$$

where for reasons of admissibility (see (3.11))

$$
\begin{aligned}
& -1 \leqq \theta_{3} \leqq 0 ; \\
& -1 \leqq \theta_{4} \leqq 0 \text { if } \theta_{1}=1 ; \\
& -1 \leqq \theta_{1} \leqq 0 \text { if } \theta_{4}=1,
\end{aligned}
$$

and where $\theta_{2}= \pm 1,(4.9)$ can be converted into the following pointwise form:

$$
\begin{aligned}
& \left.\sum_{i} K\left(t_{-i}, t_{-i+1}, \ldots ; y_{-i}, y_{-i+1}, \ldots ; \eta_{-i}, \eta_{-i+1}, \ldots ; p_{-i}\right)\right|_{t=\sigma_{l-1}}+ \\
+ & \left.\sum_{i} K\left(t_{-i}, t_{-i+1}, \ldots ; y_{-i}, y_{-i+1}, \ldots ; \eta_{-i}, \eta_{-i+1}, \ldots ; p_{-i}\right)\right|_{t=\sigma_{l}} \geqq \\
\geqq & \left.\sum_{i} K\left(t_{-i}, t_{-i+1}, \ldots ; y_{-i}, y_{-i+1}, \ldots ; \eta_{-i}+\delta \eta_{-i}, \eta_{-i+1}+\delta \eta_{-i+1}, \ldots ; p_{-i}\right)\right|_{t=\sigma_{l-1}}+ \\
+ & \left.\sum_{i} K\left(t_{-i}, t_{-i+1}, \ldots ; y_{-i}, y_{-i+1}, \ldots ; \eta_{-i}+\delta \eta_{-i}, \eta_{-i+1}+\delta \eta_{-i+1}, \ldots ; p_{-i}\right)\right|_{t=\sigma_{l}}, \quad \text { (5.2) }
\end{aligned}
$$

where $y_{-i}, y_{-i+1}, \ldots ; \eta_{-i}, \eta_{-i+1}, \ldots$ denote the "optimal" variables, and where $\delta \eta_{-i+j}=0$ for $i \neq j$. We conclude that the inequality (5.2) expresses a maximum principle for problem (3.7)-(3.12): with respect to admissible variations (which satisfy (3.11) for $1 \leqq j \leqq \mu$ and decrease $h^{j}$ for $\left.\mu+1 \leqq j \leqq v\right)$ of the control variables $\eta_{0}^{j}(j=1, \ldots, m)$ the Hamiltonian is maximal for the "optimal" control $\eta_{0}$ in the sense of (5.2).

In the next section the inequality (5.2) will be retranslated in terms of the original control variables $v_{0}, v_{1}$ with regard to the absence or presence of restrictions.

\section{Reformulation of the maximum principle}

If there are no restrictions then (3.3) reduces to 


$$
v_{0}^{k}=\eta_{0}^{k}, \quad k=1, \ldots, m
$$

in other words, there is no need to introduce new control variables.

Consequently, in the notation used thus far,

$$
H\left(t, y_{0}, y_{1}, v_{0}, v_{1} ; p\right)=K\left(t_{0}, y_{0}, y_{1}, \eta_{0}, \eta_{1} ; p\right) \text {. }
$$

In this case

$$
\begin{aligned}
& H\left(t, y_{0}, y_{1}, v_{0}, v_{1} ; p\right)+H\left(t+\tau, y_{0}, y_{1}, v_{0}, v_{1} ; p\right) \geqq \\
& \quad \geqq H\left(t, y_{0}, y_{1}, v_{0}+\delta v_{0}, v_{1} ; p\right)+H\left(t+\tau, y_{0}, y_{1}, v_{0}, v_{1}+\delta v_{1} ; p\right), \\
& T_{0} \leqq t \leqq T_{1}-\tau ; \\
& H\left(t, y_{0}, y_{1}, v_{0}, v_{1} ; p\right) \geqq H\left(t, y_{0}, y_{1}, v_{0}+\delta v_{0}, v_{1} ; p\right), \quad T_{1}-\tau<t \leqq T_{1} .
\end{aligned}
$$

In words :

if there are no restrictions, the optimal control (corresponding to the extremal under consideration) maximises the Hamiltonian in the sense of (6.1), (6.2).

In the presence of restrictions one has to consider formula (5.2) very carefully. Skipping tedious considerations we only mention that in this case too $(6.1),(6.2)$ remain valid, but in the sense that:

within the region, given by the restrictions $\phi^{k} \leqq 0, k=1, \ldots, r$ and $g^{j} \leqq 0, j=1, \ldots, v$, the optimal control variables maximize the Hamiltonian in the sense of (6.1), (6.2) for admissible variations which decrease $h^{j}, 1 \leqq j \leqq v$ at $t$, and at $t+\tau$ if $t<T_{1}-\tau$.

The inequalities (6.1) and (6.2) hold on intervals where the set of active restrictions does not alter. In the partitioning points $\tau_{l}(l=0, \ldots, \lambda)$ and the points $\tau_{l} \pm \tau$ the control variables may show a jump (see example 1 of [2], Chapter VI, where $v=v_{0}$ shows a jump in $t=1$ ).

Although the maximum principle in the form of the inequalities $(6.1),(6.2)$ is of more practical importance than the inequality (5.2), the latter will prove to be of more value for further considerations. In section 7 Lagrange multipliers will be defined using derivatives of the Hamiltonian $K$ with respect to the control variables $\eta_{0}, \eta_{1}, \ldots$. Then the analogues of the "classic" necessary conditions are easily obtained from the maximum principle (5.2).

\section{Necessary conditions}

Besides the more familiar forms of the maximum principle as derived in section 6 , it is possible to get more information from the inequality (5.2) in the form of necessary conditions for the optimal variables.

In this section we shall give some differential equations for the so-called Lagrangian of the system, defined by

$$
\begin{aligned}
& L\left(t, y_{0}, y_{1}, v_{0}, v_{1} ; p\right)=H\left(t, y_{0}, y_{1}, v_{0}, v_{1} ; p\right)+ \\
& \quad+\sum_{j=1}^{v} \alpha^{j}(t) h^{j}\left(t, y_{0}, y_{1}, v_{0}, v_{1}\right)+\sum_{k=1}^{r} \lambda^{k}(t) \phi^{k}\left(t, y_{0}, y_{1}, v_{0}, v_{1}\right) .
\end{aligned}
$$

Again only the main results are mentioned; the rather substantial derivations have been omitted (see [2] for details).

We define the functions $\alpha^{1}, \ldots, \alpha^{\mu}$ of $t \in \Delta_{l}$ by

$$
\alpha^{j}(t)=\sum_{i} \frac{\partial K}{\partial \eta_{i}^{j}}[t+i \tau], \quad j=1, \ldots, \mu
$$

It then appears that the functions $\alpha^{j}$ are non-decreasing and non positive on $\Delta_{l}, j=1, \ldots, \mu$.

Similarly, defining

$$
\alpha^{j}(t)=\sum_{i} \frac{\partial K}{\partial \eta_{i}^{j}}[t+i \tau], \quad j=\mu+1, \ldots, v,
$$


it appears that the functions $\alpha^{j}(j=\mu+1, \ldots, v)$ are nonpositive constants on $\Delta_{l}$.

Define furthermore

$$
\lambda^{j-v}(t)=\sum_{i} \frac{\partial K}{\partial \eta_{i}^{j}}[t+i \tau], \quad j=v+1, \ldots, v+q,
$$

then one can show that the functions $\lambda^{j}(j=1, \ldots, q)$ are nonpositive on $\Delta_{l}$. Taking the same definition for $\lambda^{j-v}(t), j=v+q+1, \ldots, v+r$, it follows that these are identically zero on $\Delta_{l}$.

Remark. The functions $\alpha^{1}, \ldots, \alpha^{v}$ and $\lambda^{1}, \ldots, \lambda^{r}$ defined above will appear later on to be the multipliers in the Langrangian defined in (7.1). Their properties given above are of practical importance (see also [2], chapter VI, section 3).

Finally, the sum

$$
\sum_{i} \frac{\partial K}{\partial \eta_{i}^{j}}[t+i \tau], \quad v+r+1 \leqq j \leqq m,
$$

is zero on every interval $\Delta_{l}$.

The results obtained so far will be combined in the following way. Let $l$ be an integer between 1 and $m$, not to be mixed up with the index $l$ used for the intervals $\Delta_{l}$. We multiply

$$
\alpha^{j}(t+k \tau)=\sum_{i} \frac{\partial K}{\partial \eta_{i}^{j}}[t+(i+k) \tau], \quad j=1, \ldots, v ; k=0,1, \ldots
$$

by $\frac{\partial \eta_{0}^{j}}{\partial v_{k}^{l}}[t+k \tau]$ and add; analogously, we multiply

$$
\lambda^{j-v}(t+k \tau)=\sum_{i} \frac{\partial K}{\partial \eta_{i}^{j}}[t+(i+k) \tau], \quad j=v+1, \ldots, v+r ; k=0,1, \ldots
$$

by $\frac{\partial \eta_{0}^{j}}{\partial v_{k}^{l}}[t+k \tau]$ and add; finally, we multiply

$$
\sum_{i} \frac{\partial K}{\partial \eta_{i}^{j}}[t+(i+k) \tau] \quad j=v+r+1, \ldots, m ; k=0,1, \ldots
$$

by $\frac{\partial \eta_{0}^{j}}{\partial v_{k}^{l}}[t+k \tau]$ and add. This yields

$$
\begin{aligned}
& \sum_{j=1}^{v} \sum_{k}\left[\sum_{i} \frac{\partial K}{\partial \eta_{i}^{j}}[t+(i+k) \tau]-\alpha^{j}(t+k \tau)\right] \frac{\partial \eta_{0}^{j}}{\partial v_{k}^{l}}[t+k \tau]+ \\
& +\sum_{j=v+1}^{v+r} \sum_{k}\left[\sum_{i} \frac{\partial K}{\partial \eta_{i}^{j}}[t+(i+k) \tau]-\lambda^{j-v}(t+k \tau)\right] \frac{\partial \eta_{0}^{j}}{\partial v_{k}^{l}}[t+k \tau]+ \\
& +\sum_{j=v+r+1}^{m} \sum_{k}\left[\sum_{i} \frac{\partial K}{\partial \eta_{i}^{j}}[t+(i+k) \tau]\right] \frac{\partial \eta_{0}^{j}}{\partial v_{k}^{l}}[t+k \tau]=0 .
\end{aligned}
$$

Rearranging terms and considering the nature of the delayed arguments very closely it is possible to conclude from (7.5) that, in terms of the original control variables,

$$
\begin{array}{ll}
\frac{\partial L}{\partial v_{0}^{l}}[t]+\frac{\partial L}{\partial v_{1}^{l}}[t+\tau]=0, & l=1, \ldots, m ; T_{0}<t<T_{1}-\tau ; \\
\frac{\partial L}{\partial v_{0}^{l}}[t]=0, & l=1, \ldots, m ; T_{1}-\tau<t<T_{1} .
\end{array}
$$


These two equations are the first necessary conditions derived from the maximum principle.

Since $((7.1))$

$$
L=-F+\Sigma p^{j} f^{j}+\Sigma \alpha^{j} h^{j}+\Sigma \lambda^{j} \phi^{j}
$$

we have furthermore

$$
\dot{y}_{0}^{j}=f^{j}=\frac{\partial L}{\partial p^{j}}, \quad j=1, \ldots, n .
$$

In [2] we derived a canonical counterpart of this equation in the form

$$
\begin{array}{ll}
\frac{\partial L}{\partial y_{0}^{j}}[t]+\frac{\partial L}{\partial y_{1}^{j}}[t+\tau]=-\dot{p}^{j}(t), & j=1, \ldots, n ; T_{0}<t<T_{1}-\tau ; \\
\frac{\partial L}{\partial y_{0}^{j}}[t]=-\dot{p}^{j}(t), & k=1, \ldots, n ; T_{1}-\tau<t<T_{1} .
\end{array}
$$

These are the equations which, together with (7.8), form a canonical system. They can serve to compute the adjoint variables. Under the conditions imposed the solutions of these equations are continuous functions on $\left[T_{0}, T_{1}\right]$.

Collecting all conditions to be satisfied by the optimal variables we obtain the following list (corner points of state- and adjoint variables have to be excluded).

$$
\left.\begin{array}{lc}
\frac{\partial L}{\partial v_{0}^{l}}[t]+\frac{\partial L}{\partial v_{1}^{l}}[t+\tau]=0, & l=1, \ldots, m ; T_{0}<t<T_{1}-\tau ; \\
\frac{\partial L}{\partial v_{0}^{l}}[t]=0, & l=1, \ldots, m ; T_{1}-\tau<t<T_{1} ;
\end{array}\right\}
$$

On every interval $\Delta_{l}(1 \leqq l \leqq \lambda)$ :

$$
\begin{aligned}
& \left.\begin{array}{l}
g^{j}=0 \\
\alpha^{j} \leqq 0 \text { and nondecreasing }
\end{array}\right\} j=1, \ldots, \mu ; \\
& g^{j}<0 \\
& \alpha^{j} \leqq 0 \text { and constant } \\
& \} j=\mu+1, \ldots, v \\
& \left.\begin{array}{l}
\phi^{j}=0 \\
\lambda^{j} \leqq 0
\end{array}\right\} j=1, \ldots, q \\
& \left.\begin{array}{l}
\phi^{j}<0 \\
\lambda^{j}=0
\end{array}\right\} j=q+1, \ldots, r
\end{aligned}
$$

The optimal quantities $v_{0}^{l}, v_{1}^{l}, y_{0}^{i}, y_{1}^{i}, p^{i}, \alpha^{j}$ and $\lambda^{j}$ satisfy, by definition, the equations (7.14)-(7.15) and the maximum principle given in section 6 .

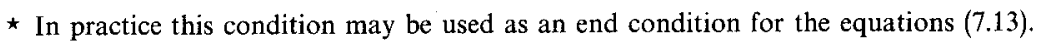


Remark. It can be deduced from the derivations of these results that the theory can immediately be extended to problems involving variables with lags of the type $\tau, 2 \tau, 3 \tau, \ldots$.

Remark 2. The conditions (7.14) may be put in the form

$$
\begin{aligned}
& \dot{\alpha}^{j}(t) g^{j}(t)=0, \quad j=1, \ldots, v, T_{0} \leqq t \leqq T_{1} ; \\
& \lambda^{j}(t) \phi^{j}(t)=0, \quad j=1, \ldots, r, T_{0} \leqq t \leqq T_{1} .
\end{aligned}
$$

and

$$
\begin{array}{ll}
\alpha^{j}(t) \leqq 0, & j=1, \ldots, v, T_{0} \leqq t \leqq T_{1} \\
\lambda^{j}(t) \leqq 0, & j=1, \ldots, r, T_{0} \leqq t \leqq T_{1} .
\end{array}
$$

Remark 3. In [2], extensions to the Hamilton-Jacobi equation and variable end point problems are considered. In particular, transversality conditions are given for free end point problems with delay.

Moreover, examples are worked out in detail.

\section{Conclusions}

For optimal centrol problems involving state- and control restrictions and time delay, necessary conditions for optimality can be derived from the maximum principle. This requires a careful analysis of the nature of the delayed arguments. Moreover, a particular choice of admissible variations of the control variables is needed to obtain the maximum-principle in pointwise form. From this, however, straight-forward analysis leads to a multiplier rule for the present type of control problems. The theory given can easily be extended to related problems, e.g. to free end point problems, as given in ref. [2].

\section{REFERENCES}

[1] R. B. Asher and H. R. Sebesta, Optimal control of systems with state-dependent time delay, Int. J. Control, 14,2 (1971) 353-365.

[2] J. F. Frankena, Optimal control problems with delay, Thesis, 1972, Twente Univ. of Techn., Enschede, Netherlands.

[3] A. Halanay, Optimal controls for systems with time lag, SIAM J. Control, 6, 2 (1968) 215-234.

[4] M. R. Hestenes, Calculus of variations and optimal control theory, Wiley and Sons (1966).

[5] Sheng-Chao Huang, Optimal control problems with retardations and restricted phase coordinates, JOTA, 3, 5 (1969) 316-360.

[6] D. K. Hughes, Contributions to the theory of variational and optimal control problems with delayed argument, Thesis (1967) Un. Oklahoma, U.S.A.

[7] D. K. Hughes, Variational and optimal control problems with delayed argument, JOTA, 2, 1 (1968) 1-14.

[8] R. Nottrot, Optimal control; on Pontryagin's maximum principle, Mathematical Comm. TH Twente, 5, 6 (1970) $1-11$.

[9] L. S. Pontryagin, V. G. Boltyankskii, R. V. Gamkrelidze and E. F. Mishenko, The mathematical theory of optimal Processes, Interscience Publishers (1963).

[10] L. D. Sabbagh, Variational systems with lags, JOTA, 3, 1 (1969) 34-51.

[11] R. Timman, Optimization theory for ordinary differential equations, J. Engineering Math., 1 (1967) $159-185$. 